\title{
Graphene Unit Cell Imaging by Holographic Coherent Diffraction
}

\author{
Jean-Nicolas Longchamp, Tatiana Latychevskaia, Conrad Escher, and Hans-Werner Fink \\ Physik Institut der Universität Zürich, Winterthurerstrasse 190, CH-8057 Zürich, Switzerland
}

(Received 7 February 2013; published 18 June 2013)

\begin{abstract}
We have imaged a freestanding graphene sheet of $210 \mathrm{~nm}$ in diameter with $2 \AA$ resolution by combining coherent diffraction and holography with low-energy electrons. The entire sheet is reconstructed from a single diffraction pattern displaying the arrangement of 660.000 individual graphene unit cells at once. Given the fact that electrons with kinetic energies of the order of $100 \mathrm{eV}$ do not damage biological molecules, it will now be a matter of developing methods for depositing individual proteins onto such graphene sheets.
\end{abstract}

DOI: 10.1103/PhysRevLett.110.255501

PACS numbers: 61.48.Gh, 42.30.Rx, 61.05.jp

Despite the vast amount of structural information data on molecules made available by crystallography, a strong desire for imaging just one individual molecule is emerging. It would allow observing different molecular conformations that remain undiscovered as long as averaging is involved. For a meaningful contribution to structural biology, any such tool for single molecule imaging has to exhibit a resolution power of at least $2 \AA$, which has not been achieved so far. The strong inelastic scattering cross section of $\mathrm{x}$ rays and high-energy electrons as employed in conventional electron microscopes inhibits accumulating a signal-to-noise ratio record before the molecule is destroyed. While future X-ray Free Electron Lasers with drastically enhanced brightness and reduced pulse duration time might eventually attain the goal of single molecule imaging [1], the current and foreseeable state of the art in $\mathrm{X}$-ray Free Electron Lasers performance requires averaging over at least $1 \times 10^{6}$ molecules to achieve atomic resolution [2]. With electrons of $200 \mathrm{keV}$ kinetic energy, radiation damage is less severe and it has been possible to image an individual carbon nanotube with atomic resolution using coherent diffraction [3]. With low-energy electrons in the range of $100 \mathrm{eV}$ molecules as fragile as DNA [4] remain unperturbed even after an exposure to a total dose of at least 5 orders of magnitude larger [5,6] than the permissible dose in x-ray or high-energy electron imaging $[7,8]$. However, the recent holographic imaging of a single ferritin molecule using such low-energy electrons exhibits a resolution barely below one nanometer, not sufficient to reveal any interesting structural detail of this protein [6]. Below we show that in using a highly transparent support and a combination of holography and coherent diffraction imaging [9] a resolution of $2 \AA$ is achieved. For coherent diffraction imaging using low-energy electrons, a parallel

Published by the American Physical Society under the terms of the Creative Commons Attribution 3.0 License. Further distribution of this work must maintain attribution to the author(s) and the published article's title, journal citation, and DOI. wave front is needed and is formed by a micromachined electron lens placed in front of the electron point source [10,11].

Since freestanding graphene is highly transparent for low-energy electrons [12,13] it is possible to perform holography and coherent diffraction with the same sample. In the following, we show that a planar low-energy electron wave front provides a high-resolution coherent diffraction pattern of a circular $210 \mathrm{~nm}$ diameter graphene sample exhibiting diffraction spots corresponding to $2.13 \AA$. Since the phase of the scattered wave is not known a priori, we also record a hologram of the very same sample and, hence, gain coarse information about the phase distribution. The combination of this initial phase distribution with the single high-resolution diffraction pattern [9] allows us to iteratively reconstruct the entire $210 \mathrm{~nm}$ diameter graphene sheet displaying about 660.000 unit cells at once. A schematic of the experimental setup with its two modes of operation, coherent diffraction and holography, is displayed in Fig. 1.

Ultraclean graphene was prepared by the platinum metal catalysis method [14], resulting in a freestanding graphene sheet placed over a $210 \mathrm{~nm}$ diameter bore ion milled through a platinum covered SiN membrane of $50 \mathrm{~nm}$ thickness. An electron hologram of such a freestanding graphene sheet is shown in Fig. 2 together with a coherent diffraction pattern of the very same sample. In both experimental schemes, the source of the coherent electron beam consists of a sharp $\mathrm{W}(111)$ tip that is driven by a 3-axis piezomanipulator with nanometer precision. In the holographic mode (Fig. 1) the tip is brought as close as $380 \mathrm{~nm}$ to the sample and in the coherent diffraction mode, the distance between the electron source and the micro-lens is approximately 8 microns. In order to collimate the divergent electron beam originating from the electron source, an electrical potential difference of typically $50 \mathrm{~V}$ is applied between the two electrodes of the micro lens. The sample is positioned into the parallel beam approximately 200 microns behind the lens with the help of a nanopositioner with three translational and one rotational axis. 


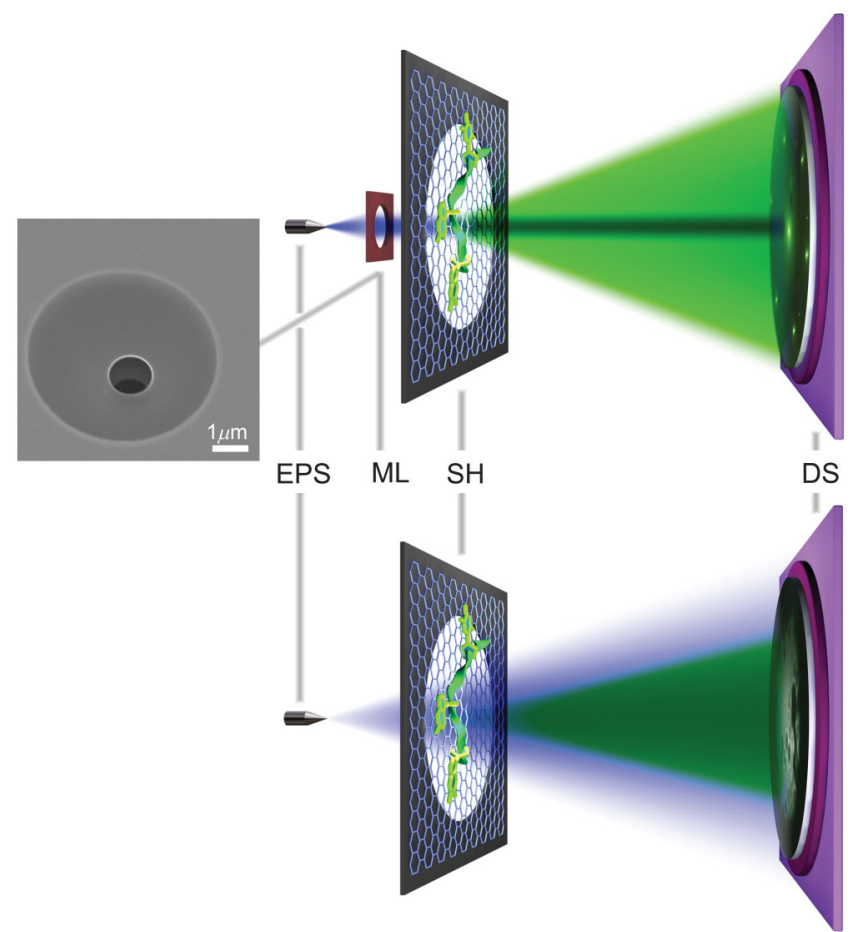

FIG. 1 (color). Experimental setup for coherent diffraction and holographic imaging of freestanding graphene. An electron point-source (EPS) emits a spherical electron wave. For coherent diffraction imaging (top), a micro-lens (ML) with a bore of 1 micron (see inset showing a SEM image of the lens) is employed to form a parallel beam impinging onto the freestanding graphene mounted onto a sample holder $(\mathrm{SH})$. The diffraction patterns, respectively, the holograms (bottom), are recorded at a $68 \mathrm{~mm}$ distant detector system, consisting of a $75 \mathrm{~mm}$ diameter microchannel plate, followed by a phosphorous coated fiberoptic plate and an $8000 \times 6000$ pixels CCD chip.

The resulting diffraction pattern is collected at a $68 \mathrm{~mm}$ distant electron detector of $75 \mathrm{~mm}$ in diameter (Fig. 1) and captured by an $8000 \times 6000$ pixels CCD chip. The outermost diffraction spots in the pattern displayed in Fig. 2 correspond to $2.13 \AA$ as determined by the de Broglie wavelength of the electrons and the angle under which they appear on the detector. Because of the fact that the initial phase distribution is directly available from the hologram and that the diffraction pattern is sufficiently fine sampled, freestanding graphene can be reconstructed with a resolution of $2 \AA$.

This is done in the following manner. The experimental records, 16-bit TIFF images with $8000 \times 6000$ pixels in size, are first cropped to $6000 \times 6000$ pixels. The multichannel plate grid image is numerically filtered out of the hologram by blocking the characteristic peaks in the Fourier domain. Thereafter, the hologram is normalized by division with the background image [15] and iteratively reconstructed [16,17]. A twin-image suppressed reconstruction is already achieved after 6 iterations.
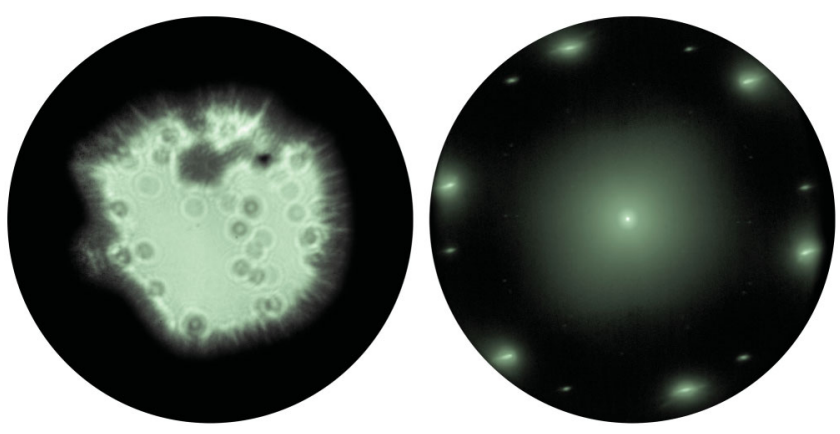

FIG. 2 (color). Low-energy electron hologram and coherent diffraction pattern of freestanding graphene. Left: Hologram of freestanding graphene placed over a $210 \mathrm{~nm}$ diameter bore in a Pt covered SiN membrane, recorded with $58 \mathrm{eV}$ electrons. Right: $236 \mathrm{eV}$ electrons coherent diffraction pattern of the very same sample. Two sets of sixfold symmetric diffraction patterns, indicating the presence of two different orientations in the graphene layer, are apparent. The intensity ratio of the two sets of diffraction spots reflects the occurrence of the two different orientations. The width of the diffraction spots is related to the finite size of the sample.

In order to build up a high dynamic range image, three diffraction patterns taken with different exposure times are combined appropriately. Based on the fact that the diffraction pattern of a real-valued object must be centrosymmetric, the experimental data are symmetrized for further enhancement of the signal-to-noise ratio [18]. Accordingly, the intensity of each pair of centrosymmetric pixels is set to their averaged intensity. Next, the diffraction pattern is transformed to spherical coordinates and multiplied with an apodization function to smoothen the transition to zero at the edges. In order to enhance the number of pixels per unit cell in the reconstruction, the diffraction pattern is zero padded to $10.000 \times 10.000$ pixels. Accordingly, the hologram reconstruction is also resampled to $10.000 \times 10.000$ pixels. Recently, it has been recognized that there exists a mathematical relationship between a diffraction pattern and a hologram; in fact, the Fourier transform of a hologram is isomorphic to the coherent diffraction pattern of the same object [9]. Based on this notion, the overexposed region in the center of the diffraction pattern is replaced by the corresponding 100 pixels in radius region of the squared amplitude of the Fourier transform of the hologram [9]. The iterative routine for the reconstruction is based on the Fienup algorithm [19]. The phase distribution used for the first iteration is provided by the phase of the Fourier transform of the hologram reconstruction. The constraint applied in the object domain is positive absorption [16]. After the 50th iteration, a mask is applied to set the transmission function outside the graphene covered bore to zero [17]. In the detector plane the constraint is just the basic physics concept that the amplitude must be equal to the square root of the measured intensity. After 100 iterations, the 

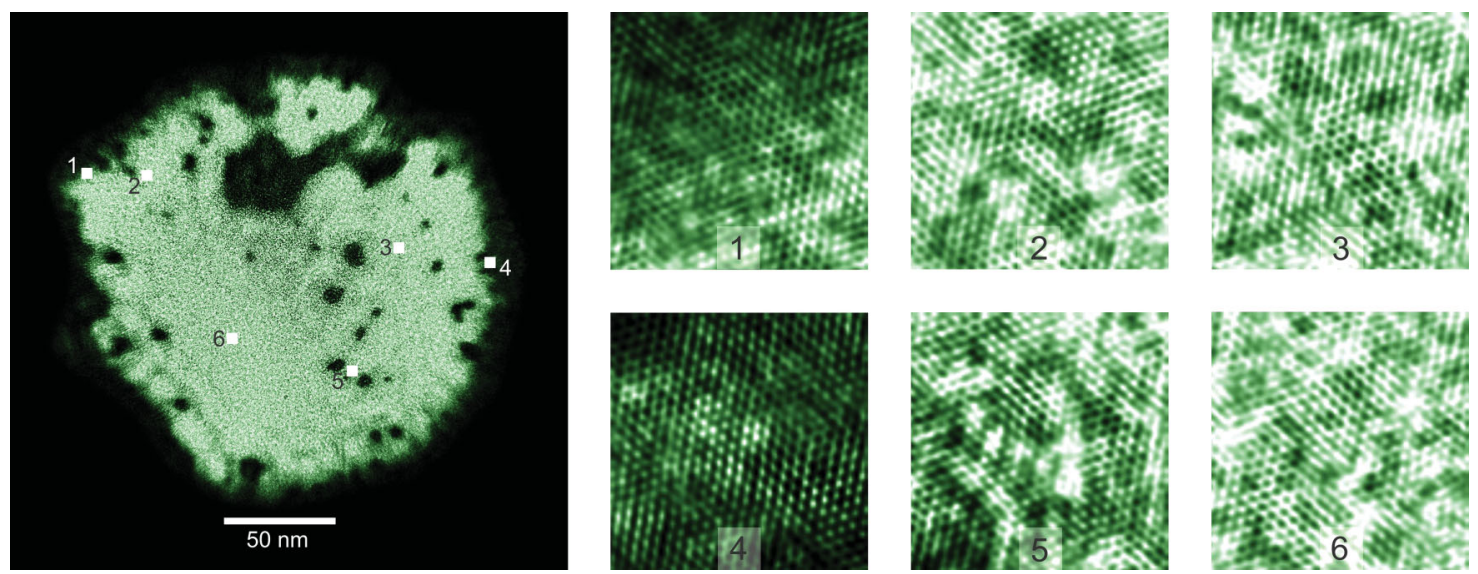

FIG. 3 (color). Reconstruction of a $210 \mathrm{~nm}$ diameter freestanding graphene sheet with $2 \AA$ resolution. Left: reconstruction of the entire $210 \mathrm{~nm}$ diameter graphene sheet labeled with squares of $5 \times 5 \mathrm{~nm}^{2}$. Right: detailed view of the marked region in the left panel.

entire $210 \mathrm{~nm}$ diameter freestanding graphene sheet is recovered with $2 \AA$ resolution sufficient to display the roughly 660.000 graphene unit cells (Fig. 3, left). Since there is no way to print or display the entire reconstructed region of $10.000 \times 10.000$ pixels here, we have cut out and magnified some selected square areas of $5 \mathrm{~nm}$ side length [20]. The graphene unit cell is clearly apparent along with defects and domain boundaries present in the graphene sheet (Fig. 3, right). This is anticipated since the graphene sample is CVD grown on a polycrystalline copper substrate. In Fig. 4, we show another cut-out region with a size of $7.4 \times 7.4 \mathrm{~nm}^{2}$ where we have marked some apparent lattice defects in red.

To conclude, we have demonstrated that low-energy electrons allow damage-free imaging of a $210 \mathrm{~nm}$ diameter circular graphene sheet with $2 \AA$ resolution. A single diffraction pattern, if sampled at a sufficiently high rate, provides a real space image of more than $0.5 \times 10^{6}$ unit cells at once. Regarding freestanding graphene as support for molecules to be analyzed, it will now be an immediate challenge to place a single protein onto such sample carrier and image it with the $2 \AA$ resolution.

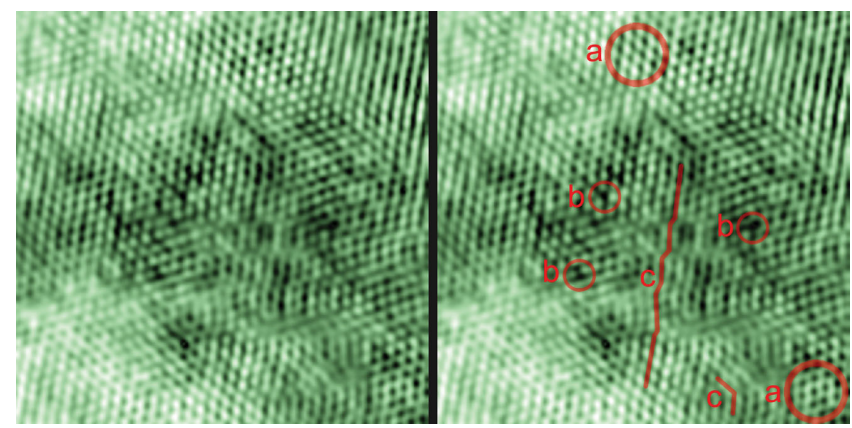

FIG. 4 (color). Two identical copies of a $7.4 \times 7.4 \mathrm{~nm}^{2}$ region of the freestanding graphene sheet exhibiting various defects of the CVD grown graphene, marked in the copy at right with red circles, respectively lines: (a) regions displaying perfectly arranged unit cells, (b) point defects, and (c) domain boundaries.
J.-N.L. performed the experiments and acquired the holograms and diffraction patterns. T. L. developed the numerical routines and performed the reconstruction of holograms and diffraction patterns. C. E. and H.-W. F. contributed to the design of the experimental setup and to sample preparation. All authors jointly drafted the manuscript. The work presented here has been financially supported by the Swiss National Science Foundation (SNF).

[1] R. Neutze, R. Wouts, D. van der Spoel, E. Weckert, and J. Hajdu, Nature (London) 406, 752 (2000).

[2] J. W. Miao, H. N. Chapman, J. Kirz, D. Sayre, and K. O. Hodgson, Annu. Rev. Biophys. Biomol. Struct. 33, 157 (2004).

[3] J. M. Zuo, I. Vartanyants, M. Gao, R. Zhang, and L. A. Nagahara, Science 300, 1419 (2003).

[4] H.-W. Fink, H. Schmid, E. Ermantraut, and T. Schulz, J. Opt. Soc. Am. A 14, 2168 (1997).

[5] M. Germann, T. Latychevskaia, C. Escher, and H.-W. Fink, Phys. Rev. Lett. 104, 095501 (2010).

[6] J.-N. Longchamp, T. Latychevskaia, C. Escher, and H.-W. Fink, Appl. Phys. Lett. 101, 093701 (2012).

[7] M. van Heel et al., Q. Rev. Biophys. 33, 307 (2000).

[8] J. Frank, Annu. Rev. Biophys. Biomol. Struct. 31, 303 (2002).

[9] T. Latychevskaia, J.-N. Longchamp, and H.-W. Fink, Opt. Express 20, 28871 (2012).

[10] H.-W. Fink, Phys. Scr. 38, 260 (1988).

[11] E. Steinwand, J.-N. Longchamp, and H.-W. Fink, Ultramicroscopy 110, 1148 (2010).

[12] J. Y. Mutus, L. Livadaru, J. T. Robinson, R. Urban, M. H. Salomons, M. Cloutier, and R. A. Wolkow, New J. Phys. 13, 063011 (2011).

[13] J.-N. Longchamp, T. Latychevskaia, C. Escher, and H.-W. Fink, Appl. Phys. Lett. 101, 113117 (2012).

[14] J.-N. Longchamp, C. Escher, and H.-W. Fink, J. Vac. Sci. Technol. B 31, 020605 (2013).

[15] T. Latychevskaia and H.-W. Fink, Opt. Express 17, 10697 (2009). 
[16] T. Latychevskaia and H.-W. Fink, Phys. Rev. Lett. 98, 233901 (2007).

[17] T. Latychevskaia, P. Formanek, C. T. Koch, and A. Lubk, Ultramicroscopy 110, 472 (2010).

[18] O. Kamimura, Y. Maehara, T. Dobashi, K. Kobayashi, R. Kitaura, H. Shinohara, H. Shioya, and K. Gohara, Appl. Phys. Lett. 98, 174103 (2011).
[19] J. R. Fienup, Appl. Opt. 21, 2758 (1982).

[20] See Supplemental Material at http://link.aps.org/ supplemental/10.1103/PhysRevLett.110.255501 for this video, the graphene hexagonal structure is shown by scanning through the entire reconstruction of the freestanding graphene sheet exhibiting a total of 660.000 unit cells. 